\title{
Corrigendum
}

\section{Corrigendum to "All-ceramic or metal-ceramic tooth-supported fixed dental prostheses (FDPs)? A systematic review of the survival and complication rates. Part II: Multiple-unit FDPs"}

\author{
Bjarni Elvar Pjetursson ${ }^{a, *}$, Irena Sailer ${ }^{b}$, Nikolay Alexandrovich Makarov ${ }^{b}$, \\ Marcel Zwahlen c, Daniel Stefan Thoma ${ }^{d}$ \\ a Division of Reconstructive Dentistry, Faculty of Odontology, University of Iceland, Reykjavik, Iceland \\ $\mathrm{b}$ Division of Fixed Prosthodontics and Biomaterials, Clinic of Dental Medicine, University of Geneva, Switzerland \\ c Institute of Social and Preventive Medicine, University of Berne, Berne, Switzerland \\ $\mathrm{d}$ Clinic of Fixed and Removable Prosthodontics and Dental Material Science, University of Zurich, Zurich, \\ Switzerland
}

The authors would like to inform the readers of a needed clarification in this article:

Analyzing the difference types of tooth-supported fixed dental prosthesis (FDPs) in part II of the review, the FDPs were also divided into four material groups; metal ceramic, reinforced glass ceramics, glass infiltrated alumina and densely sintered zirconia. Seven studies reporting on 208 FDPs were included in the "reinforced glass ceramic group" and 15 studies reporting on 673 FDPs were included in the "densely sintered zirconia group".

Unfortunately, five of the included studies [1-5] reported on a patient cohort that was reported on in another publications, also included in the present review. Hence, two of the studies reporting glass infiltrated alumina FDPs and one study reporting on densely sintered zirconia FDPs should have been excluded as multiple publications in order to include only once treated patients.
After excluding these three studies the results of our new meta-analysis change as it should only include five studies reporting on 136 reinforced glass ceramic FDPs and 14 studies reporting on 574 densely sintered zirconia FDPs. The annual failure rate of reinforced glass ceramic FDPs increased from $2.31 \%$ (95\% C.I.: $1.23-4.35 \%$ ) to 3.05\% and the estimated 5-year survival rate decreased from $89.1 \%$ (95\% C.I.: $80.4-94.0 \%$ ) to $85.9 \%$ (95\% C.I.: 74.1-92.6\%). For densely sintered zirconia FDPs the changes were minimal between the initial and the revised meta-analysis. The 5 -year survival rate decreased from $90.4 \%$ (95\% C.I.: $84.8-94.0 \%$ ) to $90.1 \%$ (95\% C.I.: 83.6-94.2\%) (revised Table 3).

The relative failure rates (as presented in Table 4 of the original publication) also change. Reinforced glass ceramic FDPs now have a statistically significantly lower survival rate $(p=0.013)$ than metal ceramic FDPs (see revised Table 4). In the original publication this difference did not reach statistical

\footnotetext{
* Corresponding author at: Department of Reconstructive Dentistry, Faculty of Odontology, University of Iceland, Vatnsmyrarvegi 16, 101 Reykjavik, Iceland. Tel.: +354 5254871.

E-mail address: bep@hi.is (B.E. Pjetursson).
} 
Table 3 - Annual failure rates and survival of metal-ceramic and all-ceramic FDPs.

\begin{tabular}{|c|c|c|c|c|c|c|c|}
\hline Study & $\begin{array}{l}\text { Year of } \\
\text { publication }\end{array}$ & $\begin{array}{l}\text { Total no. } \\
\text { of FDPs }\end{array}$ & $\begin{array}{l}\text { Mean } \\
\text { follow-up } \\
\text { time }\end{array}$ & $\begin{array}{l}\text { No. of } \\
\text { failure }\end{array}$ & $\begin{array}{l}\text { Total FDPs } \\
\text { exposure } \\
\text { time }\end{array}$ & $\begin{array}{l}\text { Estimated annual } \\
\text { failure rate }{ }^{*} \text { per } 100 \\
\text { FDP years) }\end{array}$ & $\begin{array}{l}\text { Estimated survival } \\
\text { after } 5 \text { years* (in } \\
\text { percent) }\end{array}$ \\
\hline \multicolumn{8}{|l|}{ Metal ceramic } \\
\hline Svanborg et al. & 2013 & 201 & 4.6 & 7 & 915 & 0.77 & $96.2 \%$ \\
\hline Peleaz et al. & 2012 & 20 & 4.2 & 0 & 83 & 0 & $100 \%$ \\
\hline Wolleb et al. & 2012 & 76 & 5.3 & 1 & 400 & 0.25 & $98.8 \%$ \\
\hline Heschl et al. & 2013 & 28 & 6.3 & 1 & 177 & 0.56 & $97.2 \%$ \\
\hline Makarouna & 2011 & 19 & 2.5 & 1 & 48 & 2.08 & $90.1 \%$ \\
\hline Brägger et al. & 2011 & 82 & 12.1 & 2 & 992 & 0.20 & $99.0 \%$ \\
\hline Christensen and Ploeger & 2010 & 87 & 3.0 & 5 & 261 & 1.92 & $90.9 \%$ \\
\hline Boeckler et al. & 2010 & 31 & 2.7 & 1 & 84 & 1.19 & $94.2 \%$ \\
\hline Sailer et al. & 2009 & 38 & 2.7 & 0 & 104 & 0 & $100 \%$ \\
\hline De Backer et al. & 2008 & 322 & 11.4 & 72 & 3675 & 1.96 & $90.7 \%$ \\
\hline Eliasson et al. & 2007 & 51 & 4.3 & 3 & 217 & 1.38 & $93.3 \%$ \\
\hline Hochman et al. & 2003 & 49 & 6.3 & 6 & 324 & 1.85 & $91.2 \%$ \\
\hline Walton & 2002 & 515 & 7.4 & 37 & 3363 & 1.10 & $94.6 \%$ \\
\hline Napankangas et al. & 2002 & 204 & 7.6 & 7 & 1478 & 0.47 & $97.7 \%$ \\
\hline Reichen-Grad and Lang & 1989 & 73 & 6.4 & 2 & 465 & 0.43 & $97.9 \%$ \\
\hline Total & & 1796 & 7.0 & 145 & 12,586 & & \\
\hline Summary estimate $(95 \% \mathrm{CI})$ & & & & & & $1.15(0.72-1.84)$ & $94.4 \%(91.2-96.5 \%)$ \\
\hline \multicolumn{8}{|l|}{ Reinforced glass ceramic } \\
\hline Sola-Ruiz et al. & 2013 & 21 & 10.0 & 6 & 210 & 2.86 & $86.7 \%$ \\
\hline Kern et al. & 2012 & 36 & 10.1 & 4 & 363 & 1.10 & $94.6 \%$ \\
\hline Makarouna & 2011 & 18 & 4.7 & 6 & 84 & 7.14 & $70.0 \%$ \\
\hline Esquivel et al. & 2008 & 30 & 3.3 & 5 & 100 & 5.00 & $77.9 \%$ \\
\hline Marquart et al. & 2005 & 31 & 4.2 & 6 & 129 & 4.65 & $79.3 \%$ \\
\hline Total & & 136 & 6.5 & 27 & 886 & & \\
\hline Summary estimate $(95 \% \mathrm{CI})$ & & & & & & $3.05(1.55-6.01)$ & $85.9 \%(74.1-92.6 \%)$ \\
\hline \multicolumn{8}{|l|}{ Glass infiltrated alumina } \\
\hline Christensen and Ploeger & 2010 & 23 & 3.0 & 11 & 69 & 15.9 & $45.1 \%$ \\
\hline Eschbach et al. & 2009 & 65 & 4.5 & 2 & 295 & 0.68 & $96.7 \%$ \\
\hline Suarez et al. & 2004 & 18 & 3.0 & 1 & 53 & 1.89 & $91.0 \%$ \\
\hline Olsson et al. & 2003 & 42 & 6.3 & 5 & 266 & 1.88 & $91.0 \%$ \\
\hline van Steyern et al. & 2001 & 20 & 5.0 & 2 & 95 & 2.11 & $90.0 \%$ \\
\hline Soerensen et al. & 1998 & 61 & 3.0 & 7 & 165 & 4.24 & $80.9 \%$ \\
\hline Total & & 229 & 4.1 & 28 & 943 & & \\
\hline Summary estimate (95\% CI) & & & & & & $2.97(1.20-7.35)$ & $86.2 \%(69.3-94.2 \%)$ \\
\hline \multicolumn{8}{|l|}{ Densely sintered zirconia } \\
\hline Rinke et al. & 2013 & 99 & 6.3 & 19 & 627 & 3.03 & $85.9 \%$ \\
\hline Raigrodski et al. & 2012 & 20 & 4.7 & 0 & 94 & 0 & $100 \%$ \\
\hline Peleaz & 2012 & 20 & 4.2 & 1 & 83 & 1.20 & $94.2 \%$ \\
\hline Lops et al. & 2012 & 28 & 6.5 & 2 & 182 & 1.10 & $94.7 \%$ \\
\hline Sax et al. & 2011 & 57 & 7.6 & 15 & 433 & 3.46 & $84.1 \%$ \\
\hline Sorrentino et al. & 2011 & 48 & 5.0 & 0 & 240 & 0 & $100 \%$ \\
\hline Schmitt et al. & 2011 & 15 & 4.0 & 0 & 60 & 0 & $100 \%$ \\
\hline Christensen and Ploeger & 2010 & 80 & 3.0 & 14 & 240 & 5.83 & $74.7 \%$ \\
\hline Beuer et al. & 2010 & 18 & 2.9 & 1 & 53 & 1.89 & $91.0 \%$ \\
\hline Sailer et al. & 2009 & 38 & 3.2 & 0 & 121 & 0 & $100 \%$ \\
\hline Wolfart et al. & 2009 & 24 & 4.0 & 1 & 97 & 1.03 & $95.0 \%$ \\
\hline Beuer et al. & 2009 & 21 & 2.5 & 2 & 53 & 3.77 & $82.8 \%$ \\
\hline Edelhoff et al. & 2008 & 22 & 3.1 & 0 & 69 & 0 & $100 \%$ \\
\hline Molin and Karlsson & 2008 & 19 & 5.0 & 0 & 95 & 0 & $100 \%$ \\
\hline Tinschert et al. & 2008 & 65 & 3.1 & 0 & 202 & 0 & $100 \%$ \\
\hline 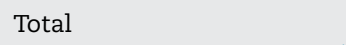 & & 574 & 4.6 & 55 & 2649 & & \\
\hline Summary estimate $(95 \% \mathrm{CI})$ & & & & & & $2.08(1.20-3.58)$ & $90.1 \%(83.6-94.2 \%)$ \\
\hline Overall results & & 2735 & 6.2 & 255 & 17,063 & & \\
\hline Summary estimate $(95 \% \mathrm{CI})$ & & & & & & $1.49(1.11-2.01)$ & $92.8 \%(90.4-94.6 \%)$ \\
\hline
\end{tabular}




\begin{tabular}{|c|c|c|c|c|c|c|c|}
\hline Type of FDPs & $\begin{array}{l}\text { Total } \\
\text { number } \\
\text { of FDPs }\end{array}$ & $\begin{array}{l}\text { Total FDPs } \\
\text { exposure } \\
\text { time }\end{array}$ & $\begin{array}{l}\text { Mean FDPs } \\
\text { follow-up } \\
\text { time }\end{array}$ & $\begin{array}{l}\text { Estimated } \\
\text { annual failure } \\
\text { rate }^{*}\end{array}$ & $\begin{array}{l}5 \text { year survival } \\
\text { summary estimate } \\
(95 \% \text { CI })\end{array}$ & $\begin{array}{l}\text { Relative } \\
\text { failure rate }\end{array}$ & $p$-Value ${ }^{* *}$ \\
\hline Metal-ceramic & 1796 & 12,586 & 7.0 & $1.15(0.72-1.84)$ & $94.4 \%$ (91.2-96.5\%) & 1.00 (Ref.) & \\
\hline Reinforced glass ceramic & 136 & 885 & 6.5 & $3.05(1.55-6.01)$ & $85.9 \%$ (74.1-92.6\%) & $2.65(1.23-5.69)$ & 0.013 \\
\hline Glass- infiltrated alumina & 229 & 943 & 4.1 & $2.97(1.20-7.35)$ & $86.2 \%$ (69.3-94.2\%) & $2.58(0.99-6.69)$ & 0.052 \\
\hline Densely sintered zirconia & 673 & 3062 & 4.5 & $2.08(1.20-3.58)$ & $90.1 \%(83.6-94.2 \%)$ & $1.80(0.89-3.64)$ & 0.101 \\
\hline
\end{tabular}

\begin{tabular}{|c|c|c|c|c|c|c|c|c|c|}
\hline \multirow[t]{2}{*}{ Complication } & $\begin{array}{l}\text { Number of } \\
\text { abutments } \\
\text { or FDPs }\end{array}$ & $\begin{array}{l}\text { Estimated annual } \\
\text { complication } \\
\text { rates ( } 95 \% \text { CI) }\end{array}$ & $\begin{array}{l}\text { Cumulative } 5 \text { year } \\
\text { complication rates } \\
(95 \% \text { CI })\end{array}$ & $\begin{array}{l}\text { Number of } \\
\text { abutments } \\
\text { or FDPs }\end{array}$ & $\begin{array}{l}\text { Estimated annual } \\
\text { complication } \\
\text { rates ( } 95 \% \text { CI) }\end{array}$ & $\begin{array}{l}\text { Cumulative } 5 \text { year } \\
\text { complication rates } \\
(95 \% \text { CI) }\end{array}$ & $\begin{array}{l}\text { Number of } \\
\text { abutments } \\
\text { or FDPs }\end{array}$ & $\begin{array}{l}\text { Estimated annual } \\
\text { complication } \\
\text { rates }(95 \% \mathrm{CI})\end{array}$ & $\begin{array}{l}\text { Cumulative } \\
5 \text { year compli- } \\
\text { cation rates } \\
(95 \% \mathrm{CI})\end{array}$ \\
\hline & \multicolumn{3}{|c|}{ Overall results-all FDPs } & \multicolumn{3}{|c|}{ Metal ceramic FDPs } & \multicolumn{3}{|c|}{ Reinforced glass ceramic FDPs } \\
\hline Caries on abutments & 3351 & $0.29^{*}(0.14-2.94)$ & $1.4 \%^{*}(0.7-2.9 \%)$ & 2497 & $0.24^{*}(0.10-0.57)$ & $1.2 \%^{*}(0.5-2.8 \%)$ & 199 & $0.12^{*}(0.01-1.27)$ & $0.6 \%{ }^{*}(0.06-6.2 \%)$ \\
\hline FDPs lost due to caries & 2145 & $0.43^{*}(0.21-0.88)$ & $2.1 \%^{*}(1.1-4.3 \%)$ & 1053 & $0.54^{*}(0.24-1.22)$ & $2.7 \%^{*}(1.2-5.9 \%)$ & 118 & $0.12^{*}(0.01-1.29)$ & $0.6 \%{ }^{*}(0.06-6.2 \%)$ \\
\hline $\begin{array}{l}\text { FDPs lost due to } \\
\text { periodontal disease }\end{array}$ & 2096 & $0.23^{*}(0.10-0.54)$ & $1.2 \%^{*}(0.5-2.7 \%)$ & 1004 & $0.06^{*}(0.03-0.11)$ & $0.3 \%^{*}(0.1-0.6 \%)$ & 118 & $0.87^{*}(0.14-5.53)$ & $4.3 \%^{*}(0.7-24.1 \%)$ \\
\hline $\begin{array}{l}\text { FDPs lost due to } \\
\text { abutment tooth } \\
\text { fracture }\end{array}$ & 2107 & $0.17^{*}(0.12-0.25)$ & $0.9 \%^{*}(0.6-1.3 \%)$ & 1053 & $0.19^{*}(0.11-0.30)$ & $0.9 \%^{*}(0.6-1.5 \%)$ & 118 & $0.12^{*}(0.03-0.53)$ & $0.6 \%^{*}(0.1-2.6 \%)$ \\
\hline $\begin{array}{l}\text { Loss of abutment tooth } \\
\text { vitality }\end{array}$ & 243 & $0.44^{*}(0.11-1.80)$ & $2.2 \%^{*}(0.5-8.6 \%)$ & n.a. & n.a. & n.a. & n.a. & n.a. & n.a. \\
\hline Marginal discolorations & 253 & $3.91^{*}(1.46-10.46)$ & $17.7 \%{ }^{*}(7.0-40.7 \%)$ & 20 & $4.82^{*}(1.33-11.88)$ & $21.4 \%^{*}(6.4-44.8 \%)$ & 118 & $0.72 .^{*}(0.23-2.19)$ & $3.5 \%^{*}(1.2-10.4 \%)$ \\
\hline Framework fracture & 2640 & $0.45^{*}(0.25-0.82)$ & $2.2 \%^{*}(1.2-4.0 \%)$ & 1530 & $0.12^{*}(0.04-0.40)$ & $0.6 \%^{*}(0.2-2.0 \%)$ & 136 & $2.14^{*}(1.0-4.74)$ & $10.1 \%(4.7-21.1 \%)$ \\
\hline Ceramic fractures & 2129 & $1.56^{*}(0.85-2.86)$ & $7.5 \%$ (4.2-13.3\%) & 1305 & $1.03^{*}(0.42-2.56)$ & $5.0 \%^{*}(2.1-12.0 \%)$ & 115 & $1.63^{*}(1.46-1.82)$ & $7.8 \%^{*}(7.0-8.7 \%)$ \\
\hline Ceramic chipping & 1659 & $2.71^{*}(1.52-4.83)$ & $12.7 \%^{*}(7.3-21.4 \%)$ & 781 & $1.79^{*}(0.81-3.96)$ & $8.6 \%^{*}(4.0-18.0 \%)$ & 141 & $1.45^{*}(0.70-3.01)$ & $7.0 \%{ }^{*}(3.5-14.0 \%)$ \\
\hline Loss of retention & 1702 & $0.64^{*}(0.35-1.16)$ & $3.1 \%^{*}(1.7-5.6 \%)$ & 955 & $0.42^{*}(0.16-1.09)$ & $2.1 \%^{*}(0.8-5.3 \%)$ & 106 & $0.51^{*}(0.23-1.12)$ & $2.5 \%^{*}(1.1-5.4 \%)$ \\
\hline
\end{tabular}




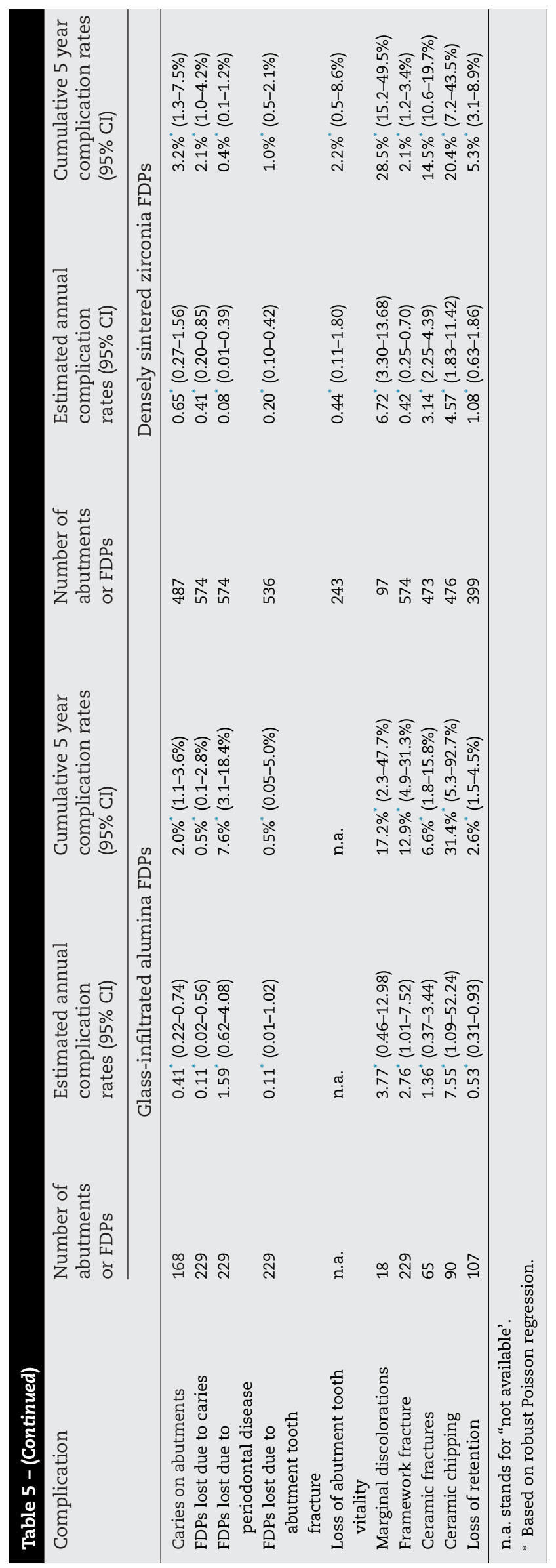

significance $(p=0.068)$. For densely sintered zirconia FDPs the difference was minor and had no influence on the relative failure rate. The main conclusion of the systematic review, that survival rates of all types of all-ceramic FDPs were lower than those reported for metal-ceramic FDPs has not changed.

Excluding the multiple publications also had a slight influence on the incidence of biological and technical complications of the reinforced glass ceramic and densely sintered zirconia FDPs (see revised Table 5). The minor changes in the incidence of biological and technical complications did hardly change the results of the comparison of the complication rate of reinforced glass ceramic and densely sintered zirconia FDPs with complication rates of other types of FDS.

The authors wish to apologize for any inconvenience caused.

\section{REFERENCES}

[1] Kern T, Tinschert J, Schley JS, Wolfart S. Five-year clinical evaluation of all-ceramic posterior FDPs made of In-Ceram Zirconia. Int J Prosthodont 2012;25:622-4.

[2] Rinke S, Gersdorff N, Lange K, Roediger M. Prospective evaluation of zirconia posterior fixed partial dentures: 7-year clinical results. Int J Prosthodont 2013;26:164-71.

[3] Roediger M, Gersdorff N, Huels A, Rinke S. Prospective evaluation of zirconia posterior fixed partial dentures: four-year clinical results. Int J Prosthodont 2010;23:141-8.

[4] Wolfart S, Bohlsen F, Wegner SM, Kern M. A preliminary prospective evaluation of all-ceramic crown-retained and inlay-retained fixed partial dentures. Int J Prosthodont 2005;18:497-505.

[5] Wolfart S, Eschbach S, Scherrer S, Kern M. Clinical outcome of three-unit lithium-disilicate glass-ceramic fixed dental prostheses: up to 8 years results. Dent Mater 2009;25:e63-71. 